\title{
Cerebral astroblastoma-a rare and elusive case
}

\author{
Panda $S^{1}$, Pattanaik $R^{2}$, Bhagat $S^{3}$, Nisa $S^{4}$, Panda B. $B^{5}$, Dash $B^{6}$ \\ ${ }^{1}$ Dr. Subhasish Panda, Resident, ${ }^{2}$ Dr. Rajesh Pattanaik, Resident, ${ }^{3}$ Dr. Savitri Bhagat, Professor, ${ }^{4}$ Dr. S. Nisa, Associate \\ Professor, ${ }^{5}$ Dr. Braja Behari Panda, Associate Professor, ${ }^{6}$ Dr. Bararuchi Dash, Associate Professor. All affiliated to P.G. \\ Department of Radiodiagnosis, V.S.S. Institute of Medical Sciences and Research, Burla, Sambalpur, Odisha, India.
}

Address for Correspondence: Dr. Subhasish Panda, Email: drsubhasishpanda @ gmail.com

\begin{abstract}
Background: Astroblastoma is rare neuroglial intracranial tumor most commonly occurring in the first three decades of life. Case report: A 5 year old female child presented with intermittent headache, diplopia and recurrent seizures over a period of approximately 4 months. Her neurological examination revealed $6^{\text {th }}$ nerve palsy and papilledema. Both CT and MRI revealed a well defined enhancing solid-cystic mass in the left fronto-parietal cerebral cortex with mass effect suggestive of primitive neuroectodermal tumor. She underwent gross total resection of the lesion through craniotomy. Histopathology along with immunohistochemistry was surprisingly suggestive of astroblastoma. Conclusion: Astroblastomas are very rare and often misdiagnosed.
\end{abstract}

Key words: Computerized tomography, Magnetic resonance imaging, Supratentorial.

\section{Introduction}

Astroblastomas are well circumscribed intracranial tumors of neuroglial origin with a very rare incidence with approximately 230 cases reported globally till date $[1,2]$. They are most commonly found in the first three decades of life. Literature reports that astroblastoms comprise 0.5 to $2.8 \%$ of primary gliomas. They mimic astrocytoma, ependymoma, primitive neuroectodermal tumor \& dysembryoplastic neuroepithelial tumor radiologically and hence are frequently underdiagnosed [2]. They almost always located supratentorially in the brain parenchyma, with few exceptions. They are situated peripheral in the cerebral cortex, comprising of both solid and cystic components [3]. Early diagnosis followed by gross total resection of the tumor with subsequent radiotherapy and/or chemotherapy for the high grade lesions is advocated.

\section{Case Report}

A 5 year old female child presented to the Department of Medicine of our Institution with intermittent headache, diplopia and recurrent seizures over a period of approximately 4 months. Her neurological examination revealed $6^{\text {th }}$ nerve palsy and papilledema.

She was referred to the Department of Radiodiagnosis where she was subjected to computerized tomography (CT) scan of the brain which revealed a mixed solid-cystic mass lesion of size 90 x85x75 mm over left frontal and parietal lobe. The solid component was located peripherally in a superolateral location. The cystic component showed multiple internal septation (Fig 1). IV Contrast CT Scan image depicted heterogenous enhancement. The differential diagnoses given based on CT imaging were primitive neuro-ectodermal tumor (PNET) and dysembryoplastic infantile ganglioglioma (DIG).

Magnetic Resonance Imaging (MRI) was subsequently performed where the cystic component of the space occupying lesion demonstrated high signal on $\mathrm{T} 2 \mathrm{~W}$ sequence. The solid component had hypo-to-isointense signal on $\mathrm{T} 1 \mathrm{~W}$ and

Manuscript received $25^{\text {th }}$ October 2016

Reviewed: $6^{\text {th }}$ November 2016

Author Corrected: $18^{\text {th }}$ November 2016

Accepted for Publication $30^{\text {th }}$ November 2016 
mixed iso and hyperintensity on T2W sequence. On IV contrast administration, solid component as well as the wall and septations of the cystic component were noted to take enhancement. Mass effect with subfalcine herniation and midline shift of around $15 \mathrm{~mm}$ was noted to the right, with dilatation of contralateral ventricle (Fig 2). Post MRI diagnosis was PNET.

The patient underwent gross total resection of the lesion through left parietal craniotomy (Fig 3) and biopsy specimen was obtained (Fig 4). Histopathological analysis revealed a moderately cellular and vascular tumour composed of round, oval to elongated irregular cells with round to oval vesicular nuclei and pale, eosinophilic cytoplasm arranged around hyalinised vessel walls suggestive of Astroblastoma (Fig 5). Immunohistochemistry revealed position reaction to GFAP (Fig 6), S-100, Vimentin and negativity to EMA. INI was positive in a moderate number of tumor cells.

The immediate postoperative period was uneventful.
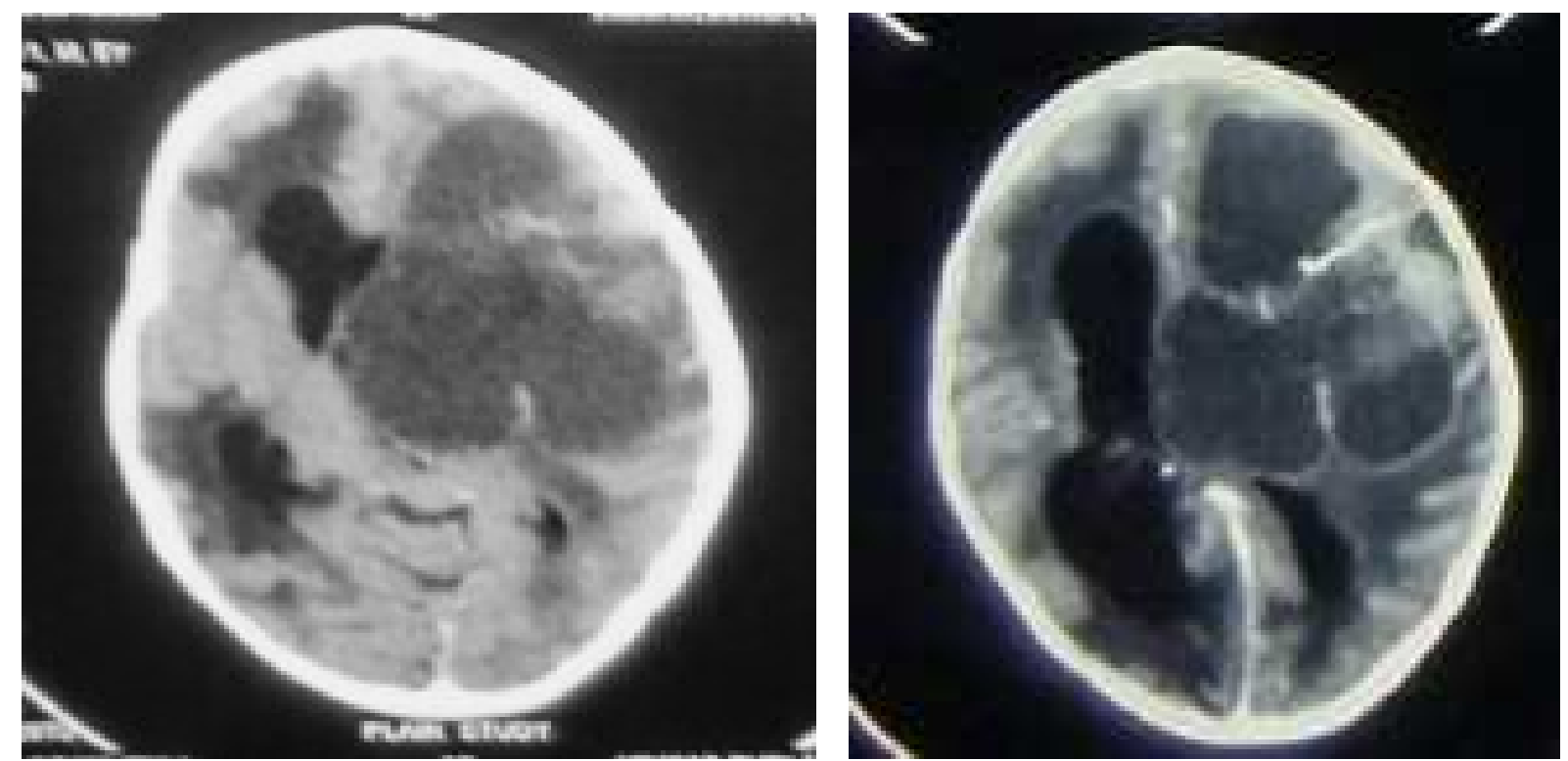

Fig-1: CT Scan - Plain and Post Contrast Images showing the intracranial tumor with solid and cystic components and its enhancement pattern, mass effect and subfalcine herniation
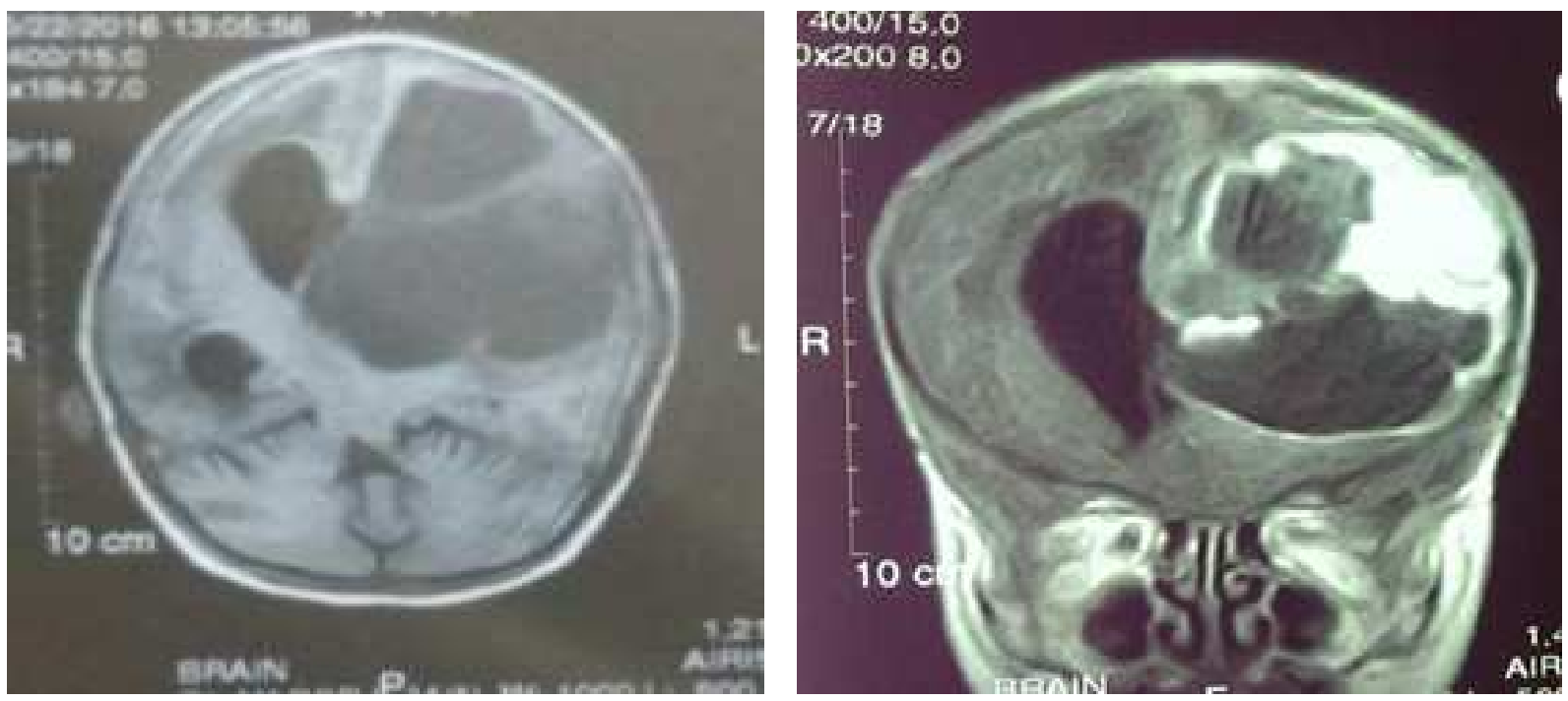

Fig-2: MRI - Plain Axial and Coronal Post Gadolinium Contrast T1 W Images showing enhancement of solid component and mural enhancement of the cystic component of the tumor. 


\section{Case Report}

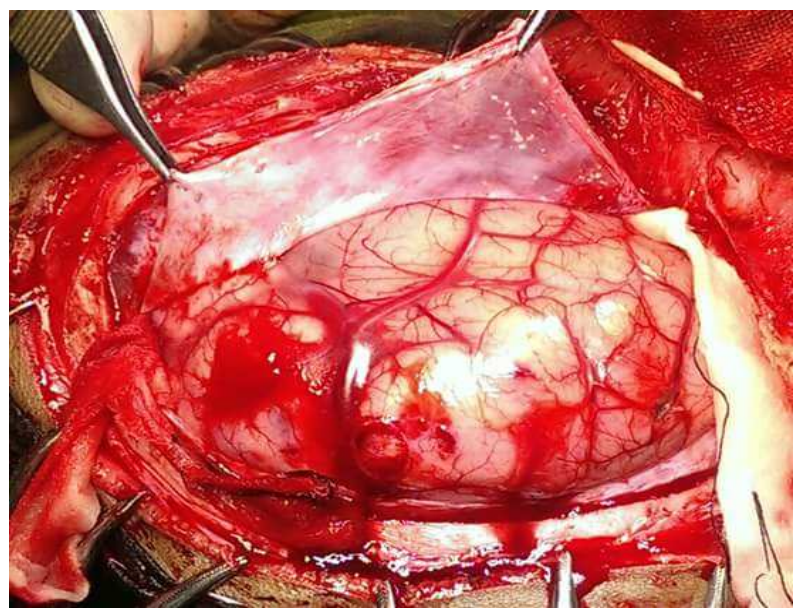

Fig 3(a): Gross Total Resection of Tumor

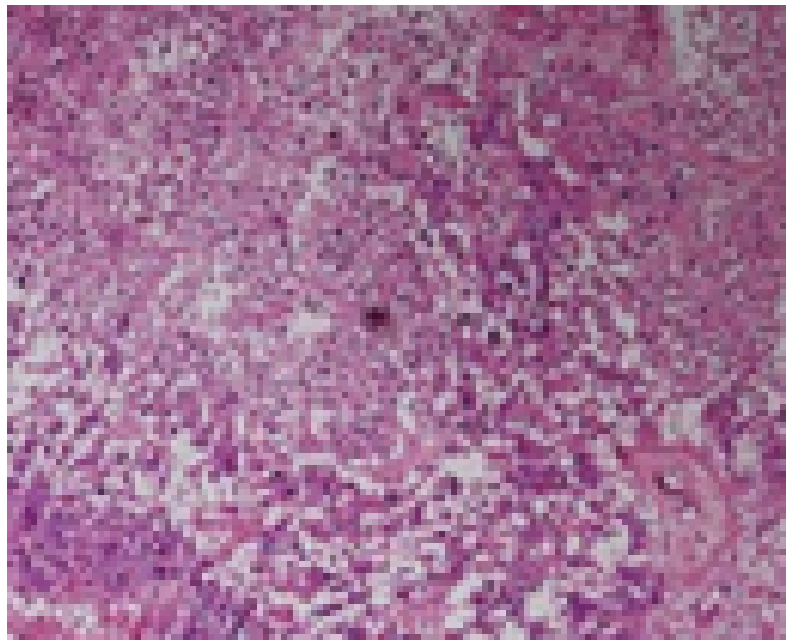

Fig 4(a): Histopathological picture

\section{Discussion}

Astroblastomas are rare neuroglial tumors with approximately 230 cases reported in literature ever since they were first described by Bailey and Cushing and later characterized by Bailey and Bucy [1]. Astroblastomas are well circumscribed intracranial tumors of glial origin [2]. They most commonly affect children and young adults [4]. Although there is no significant gender preponderance, incidence is more common in females [2,5]. Presentation is usually that of increased intracranial pressure. Symptoms of headache, seizures and focal neurological deficits are most common. The presence of a large hemispheric circumscribed solid and cystic mass in a supratentorial location is highly suggestive of its diagnosis [2]. However, some rare locations namely in the cerebellum, brainstem, corpus callosum, hypothalamus, and the ventricular system have also been reported [6]. Our case exhibited all the above features.

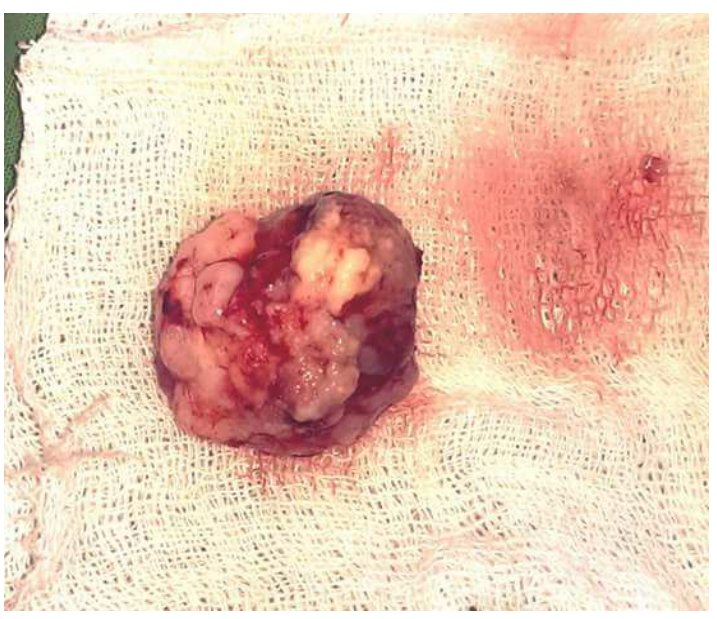

Fig 3(b): The Biopsy

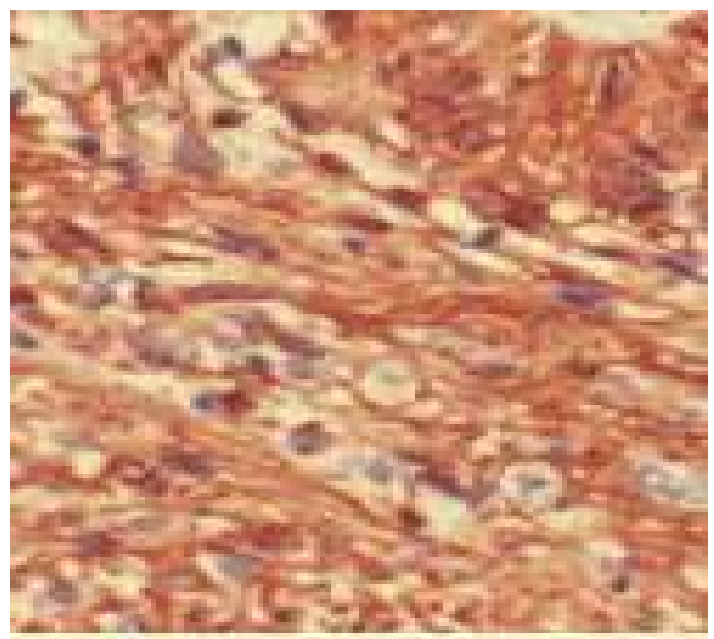

4(b): Immunohistochemistry positive for GFAP

Imaging features on computerised tomography of astroblastoma reveal a lobulated mass with solid and cystic components taking heterogenous enhancement on IV contrast administration. The solid component appears isodense to mildly hyperdense on plain study. The patient's lesion was within the ambit of these findings.

On Magnetic Resonance Imaging, the solid component of the mass usually appears isointense on $\mathrm{T} 1 \mathrm{~W}$ image and hypointense on $\mathrm{T} 2 \mathrm{~W}$ with respect to the gray matter. Post contrast T1W images typically reveal rim enhancement of the cystic component and heterogenous enhancement of the solid component. Significant perilesional edema proportionate to the size of the tumor is usually absent [2]. However, in our case homogenous enhancement of the solid component of the neoplasm was seen. 
Although the name astroblastoma suggests astrocytic origin, histologically it resembles ependymoma more than astrocytoma. Astroblastomas are defined histologically by the presence of perivascular pseudorosettes and prominent perivascular hyalinization [6]. Radiation of astrocytic cell processes towards a central hyalinisation is its characteristic [2]. In our patient the arrangement of tumor cells around blood vessels was akin to that of pseudorosettes.

There was moderate vascular endothelial proiliferation and mitotic activity, hence pathological diagnosis of astroblastoma (WHO Grade IV) was made. Astroblastomas are generally immunopositive for GFAP, S-100, vimentin and negative for EMA according to Pizer BL et al which was just the case in our patient [7].

These tumors are easily misdiagnosed as they mimic ependymoma, primitive neuroectodermal tumor \& atypical rhabdoid-teratoid tumor radiologically $[2,6]$.

The treatment advocated for astroblastomas include radical surgical resection and in cases where complete excision is not possible, chemotherapy followed by radiotherapy is advocated [8]. In our case the presence of increased vascularity and moderate cellularity suggested probable high-grade of the tumor and surgical resection was performed.

\section{Conclusion}

Without doubt, astroblastoma is one of the most challenging entities to diagnose among all CNS neoplasms. As a differential diagnosis, this tumor should be kept in mind in a young patient with a circumscribed solid and cystic intracranial mass. Demographic background, imaging features and histopathological follow up are essential in diagnosing this rare tumor.

\section{Funding: Nil, Conflict of interest: None.} Permission of IRB: Yes

\section{References}

1. Brat DJ, Hirose Y, Cohen KJ, Feuerstein BG, Burger PC. Astroblastoma: clinicopathologic features and chromosomal abnormalities defined by comparative genomic hybridization. Brain Pathol. 2000 Jul; 10 (3):342-52.

2. Karsy M, Guan J, Jensen R, Huang LE, Colman H. The Impact of Hypoxia and Mesenchymal Transition on Glioblastoma Pathogenesis and Cancer Stem Cells Regulation. World Neurosurg. 2016 Apr;88:222-36. doi: 10.1016/j.wneu.2015.12.032. Epub 2015 Dec 25.

3. Bell JW, Osborn AG, Salzman KL, Blaser SI, Jones BV, Chin SS. Neuroradiologic characteristics of astroblastoma. Neuroradiology. 2007 Mar;49(3):203-9. Epub 2007 Jan 10.

4. Barakat MI, Ammar MG, Salama HM, Abuhashem S. Astroblastoma: Case Report and Review of Literature. Turk Neurosurg. 2016;26(5):790-4. doi: 10. 5137/ 1019-5149.JTN.9408-13.2.

5. Janz C, Buhl R. Astroblastoma: report of two cases with unexpected clinical behavior and review of the literature. Clin Neurol Neurosurg. 2014 Oct;125:11424.doi:10.1016/j.clineuro.2014.07.013.Epub2014Jul 21.

6. Arcipreste AA, Felipe Padilla Vazquez, Ricardo Ramirez Aguilar, Castruita UO, Rafael Mendizabal Guerra. High-grade astroblastoma in a child: Report of one case and review of literature. Surg Neurol Int. 24Jul-2014; 5 : 111.

7. Pizer BL, Moss T, Oakhill A, Webb D, Coakham HB. Congenital astroblastoma: An immunehistochemical study. Case report. J Neurosurg. 1995; 83:550-5.

8. Salvati M, DElia A, Brogna C,Antonelli M, Giangaspero F, et al. Cerebral astroblastoma: analysis of six cases and critical review of treatment options. J Neurooncol. 2009:93(3):369-378.

\section{How to cite this article?}

Panda S, Pattanaik R, Bhagat S, Nisa S, Panda B. B, Dash B. Cerebral astroblastoma-a rare and elusive case. Int J Med Res Rev 2016;4(11):1985-1988.doi:10.17511/ijmrr. 2016.i11.15. 